\title{
Stiffness of Cell Micro-Environment Guides Long Term Cell Growth in Cell Seeded Collagen Microspheres
}

\section{Shariatzadeh M*, Perrault CM, Lacroix D}

INSIGNEO Institute for in silico Medicine, Department of Mechanical Engineering, University of Sheffield, UK

Corresponding Author: Maryam Shariatzadeh, Healthcare Engineering, Centre for Biological Engineering, Holywell Park, Loughborough University, Loughborough, LE11 3TU, UK, Tel: +44 (0)114 2220156; E-mail: m.m.shariatzadeh@1boro.ac.uk

Received: 17 January 2018; Accepted: 27 January 2018; Published: 30 January 2018

\begin{abstract}
Mesenchymal stem cells are widely implicated as a cell source for tissue engineering of skeletal tissue in cell-based therapy. Physical and mechanical cues are potent controlling factors in cell differentiation and can be implemented as a guide to study cellular response, matrix production and tissue regeneration. Microspheres were produced by gelation of bovine collagen type I with concentration of $2 \mathrm{mg} / \mathrm{mL}$ and 1,000-2,000 cells per droplet. Short and long term cell viability of human embryonic stem cell-derived mesenchymal progenitors (hES-MPs) and MG-63 osteoblastic cells as well as collagen microstructure and contraction were monitored during 28 days post encapsulation (pc). Results indicated that collagen concentration, hence mechanical properties of cell's extracellular micro-environment are important in cell proliferation and differentiation. Contraction of cell-embedded microspheres was found to be vital in cell adaptation and the remodelling of their new environment. It was also found that collagen concentration of $2 \mathrm{mg} / \mathrm{mL}$ supports proliferation of hES-MPs while higher collagen concentration promoted the viability of MG-63s. Results of hES-MPs characterization in 3D soft environment and mechanically stimulated hES-MPs collagen microspheres can be used in cells/therapeutic carriers, implants in bone and cartilage healing applications. The microspheres developed in this study can also be used as a tool to build more optimised construct to transfer mechanically stimulated stem cells to the specific area of a defective bone which would add significant benefit to the field of bone regeneration.
\end{abstract}

Keywords: Human embryonic mesenchymal stem progenitors; Collagen microspheres; Mechanical forces; Collagen contraction; Tissue engineering; 3D culture 


\section{Introduction}

Cell therapy serves as the most challenging, yet potentially the most successful application of stem cells. Mesenchymal stem cells (MSCs) are defined as mesenchymal progenitor cells with capability of self-renewal and differentiation into specialized linage in response to appropriate signals [1, 2]. Using cells seeded in suitable biomaterials to mimic the in vivo biochemical and biophysical micro-environment is a typical tissue engineering strategy, which has shown promising results in treating irreparable injuries of native tissues caused by diseases [3]. Nevertheless, before stem cells based therapies can be applied in clinics, many issues need to be elucidated to gain a precise control over cell self-renewal and differentiation. In addition, a better understanding of the interplay between MSCs and their surrounding micro-environment components including: growth factors, cell-cell, and cellextracellular matrix interactions are needed to fully comprehend the cellular events [4]. Recent studies have shown the importance of mechanical cues, including the stiffness of the substrate, nano-topography of the surface, and extracellular forces, in directing stem cell fate in vitro, even in the absence of biochemical factors [5].

Stiffness and/or topography of cellular micro-environment along with physical factors such as tension can dictate stem cell fate determination. Although physical cues control MSC lineage specification by tuning the cytoskeleton, the full mechanism of how physical signals are sensed by cells and transformed into biochemical and biological signals remains unclear [6]. Numerous studies have focused intensively on the effects of chemical signals in differentiation of stem cells [7] but, the effects of physical/mechanical signals of the micro-environment on MSCs have long been neglected [8]. However, few studies provided evidence that both direct and indirect mechanical signals are important in regulating stem cell commitment. While most studies showed that compression forces can induce chondrogenic differentiation rather than osteogenesis, some studies have demonstrated the osteogenic effect of compressive loading on stem cells $[9,10]$.

The aim of cell encapsulation is to entrap functional cells within a semi-permeable matrix. A suitable matrix must be biocompatible, support cell survival and also be permeable to oxygen, to permit diffusion of nutrients and removal of toxic metabolites [11]. Suitable materials for cell encapsulation should mimic the cells extracellular matrix. Many of these materials are based on hydrogels, which are highly hydrated and composed of hydrophilic polymers that are cross-linked to form 3D networks. Hydrogels derived from natural materials such as collagen have comparable structures to the extracellular matrix of many human tissues [12].

Natural extracellular polymers such as collagen are encapsulation materials of choice since they are the most abundant scaffold protein in tissues [13]. Their specific cell-binding sites are involved in normal cell function, and they have excellent biocompatibility and negligible immunogenicity. Collagen has been used as carriers of antibiotics genes and proteins, as well as scaffolds [6]. However, because collagen has poor mechanical and shape stability, they are incompatible with current micro-encapsulation techniques, which involve vigorous mechanical disturbances including pressurized nozzle, emulsification or stirring during droplet generation [14]. 
Chan et al. [11] introduced a novel cell micro-encapsulation technique by entrapping hMSCs in a dense mesh-work of reconstructed rat tail collagen I fibres that generated self-assembled collagen/hMSC microspheres. Their approach illustrated the feasibility of utilising these microspheres for MSC delivery by investigating the microsphere injectability, the cellular growth kinetics and the migration capability in addition to their self-renewal capacity and multi-potent differentiating potential.

Therefore, our aim was to produce a soft 3D environment consisting of self-assembled collagen I fibres, able to support long term viability and proliferation of human embryonic stem cell-derived mesenchymal progenitors (hESMPs). Also to explore the effect of 3D controlled environment in encouraging osteogenic differentiation of hESMPs in the absence of external chemical and mechanical stimulation. We explored how physical properties of cell 3D micro-environment such as stiffness can influence cell growth and commitment. We also compared cellular response of undifferentiated hES-MPs with more mature cell line (MG-63 osteoblastic cells) and monitored short and long term cell viability of both cell lines in collagen microspheres.

\section{Materials and Methods}

2.1 MG-63 cell culture

MG-63 human osteocarcoma cells were cultured in media containing $\alpha$-DMEM (Lonza, UK), 10\% FBS (Labthech, USA), $1 \%$ penicillin and streptomycin and $1 \%$ L-glutamine all purchased from Sigma, UK. Cells were incubated at $37^{\circ} \mathrm{C}$ in $5 \% \mathrm{CO}_{2}$. The medium was replenished every 3 days.

\section{2 hES-MP cell culture}

hES-MP cell line (Cellartis, Sweden) were cultured on $0.1 \%$ gelatine coated surfaces and expanded and proliferated in media containing $4 \mathrm{nM}$ fibroblast growth factor-basic recombinant human (FGF- $\beta$ ) (Life technologies, USA) as recommended by manufacturer added to $\alpha$-DMEM, $10 \%$ FBS, $1 \%$ penicillin and streptomycin and $1 \% \mathrm{~L}$-glutamine. Cell passages of 3-7 were used in the experiments. Cells were incubated at $37^{\circ} \mathrm{C}$ in $5 \% \mathrm{CO}_{2}$. The medium was replenished every 2 days.

\subsection{Collagen gelation procedure and cell seeding}

Rat tail collagen type I $(3.6 \mathrm{mg} / \mathrm{mL})$ solution in $0.02 \mathrm{~N}$ acetic acid (BD, UK) and used in gelation process following BD protocol. Collagen I was neutralized by $1 \mathrm{~N} \mathrm{NaOH}$ and 10X phosphate balance solution (PBS) both purchased from Sigma, UK and diluted into final concentrations of 0.5, 1, 2 or $3 \mathrm{mg} / \mathrm{mL}$ while being placed on ice. Then the cells (hES-MPs or MG-63s) were suspended in the neutralized collagen solution to make up cell/matrix mixtures with final cell densities of $5 \times 10^{5}$ or $1 \times 10^{6} \mathrm{cell} / \mathrm{mL}$. The volume of each substance and the number of cells required per droplet was calculated according to the collagen manufacturer protocol. The same procedure was repeated for bovine collagen I solution with a concentration of $5 \mathrm{mg} / \mathrm{mL}$. 


\begin{tabular}{|l|l|l|l|}
\hline COLLAGEN(MG/ML) & $\mathbf{5} \times \mathbf{1 0}{ }^{\mathbf{5}} \mathbf{C E L L S} / \mathbf{M L}$ & $\mathbf{1} \times \mathbf{1 0}{ }^{\mathbf{6}} \mathbf{C E L L S} / \mathbf{M L}$ & $\mathbf{5} \times \mathbf{1 0}{ }^{\mathbf{6}} \mathbf{C E L L S} / \mathbf{M L}$ \\
\hline $5 \mu \mathrm{L}$ droplet & & & \\
\hline 0.5 & 1900 & 3800 & 19000 \\
\hline 1 & 1545 & 3090 & 15450 \\
\hline 1.5 & 1195 & 2390 & 11951 \\
\hline 2 & 842 & 1685 & 8424 \\
\hline $2.5 \mu \mathrm{L}$ droplet & & & 9500 \\
\hline 0.5 & 950 & 1900 & 7725 \\
\hline 1 & 722 & 1545 & 5975 \\
\hline 1.5 & 597 & 1195 & 4212 \\
\hline 2 & 421 & 842 & \\
\hline
\end{tabular}

Table 1: Number of cells/droplet with different initial cell density, collagen concentration and droplet volume used in hES-MPs and MG-63s encapsulation.

The number of seeded cells in $5 \mu \mathrm{L}$ and $2.5 \mu \mathrm{L}$ collagen microspheres with collagen concentration of $2 \mathrm{mg} / \mathrm{mL}$ and initial cell density of $1 \times 10^{6}$ cells $/ \mathrm{ml}$ and $5 \times 10^{5}$ cells $/ \mathrm{mL}$ were calculated as 1,400 and 700 respectively. For MG63 cell line, 2,800 cells with initial cell concentration of $1 \times 10^{7}$ cells $/ \mathrm{ml}$ were seeded in $5 \mu \mathrm{L}$ collagen droplets with collagen concentration of $3 \mathrm{mg} / \mathrm{ml}$. No $2.5 \mu \mathrm{L}$ droplet was made for $3 \mathrm{mg} / \mathrm{mL}$ collagen concentration (Table 1). Collagen microspheres of $5 \mu \mathrm{L}$ and $2.5 \mu \mathrm{L}$ were dispensed in a $90 \mathrm{~mm}$ diameter Petri dish covered with UVirradiated parafilm and was incubated for $45 \mathrm{~min}$ at $37^{\circ} \mathrm{C}$ and $5 \% \mathrm{CO}_{2}$ to induce gelation of collagen solution. Gelated collagen/hES-MPs or MG-63 microspheres were then gently flushed with medium from the parafilm into a separate Petri dish. Cell-seeded microspheres were maintained free-floating and the medium was replaced with fresh complete media every $48 \mathrm{~h}$.

\subsection{Collagen fibres density and microstructure}

Microstructure of cell seeded and non-seeded collagen I fibre of bovine and rat tail was compared using scanning electron microscopy (SEM) (Philips XL-20, Cambridge, UK), second-harmonic generation (SHG) (Zeiss Axioskop 2FS MOT upright laser scanning confocal microscope equipped with tunable Chameleon Ti), confocal microscopy and collagen I specific fluorescent marker; Oregon Green ${ }^{\circledR} 488$ carboxylic acid succinimidyl ester (Invitrogen, UK). A fluorescent microscope (Nikon Ti-E, UK) was then employed to identify the variability in collagen fibres organisation with and without cells and hES-MPs role in remodelling the collagen fibre micro-structure of collagen from different species. Non-seeded collagen microspheres were examined at $24 \mathrm{~h}(\mathrm{pc})$ while seeded collagen beads were assessed at days 7 and 21 pc.

\subsection{Measurement of collagen microsphere contraction}

For collagen concentration of $2 \mathrm{mg} / \mathrm{mL}$, the effect of hES-MPs and MG-63 cell line and collagen I sources from two species of bovine and rat tail on the contraction of collagen gel were compared with each other at $8 \mathrm{~h}$, day $2,3,5,7$, 
9 and 11 post encapsulation. For each time point, 12 collagen microspheres were selected and bead diameters were measured to compare collagen gel contractibility using ImageJ software.

2.6.1 Short and long term cell viability: Cell collagen microspheres with different collagen concentration and cell density were stained with $200 \mu \mathrm{L}$ of $2 \mathrm{mM}$ calcein AM in dimethyl Sulfoxide (DMSO) (Life Technologies, USA) and $4 \mathrm{mM}$ ethidium homodimer-1 (Sigma, UK) in DMSO for $45 \mathrm{~min}$ away from light. The microspheres were then washed with PBS once to remove the excess fluorescent dyes. Collagen microspheres were flushed with full medium into 8 well $\mu$ slides ibiTreat (Thistle Scientific, UK) to check the cell viability and morphology at 8 h, 3,5 and 7 days pc. Stained microspheres were examined under fluorescent microscope (Nikon Ti-E) using FITC/Cy2 $(495-515 \mathrm{~nm})$ and ET Texas red $(515-635 \mathrm{~nm})$ filters for image acquisition.

2.6.2 Total DNA count: Total DNA was measured using a fluorescent Quant-iT dsDNA High-Sensitivity Assay Kit (Invitrogen, UK). Cells seeded microspheres were washed with PBS and lysed with a cocktail of cell digestion buffer consisting of: $1.5 \mathrm{M}$ Tris-HCL, $1 \mathrm{mM} \mathrm{ZnCL} 2$ and $\mathrm{MgCL} 2$ in $\mathrm{ddH}_{2} \mathrm{O}$ mixed with $1 \%$ Triton X-100 and incubated at $4^{\circ} \mathrm{C}$ overnight. Cell lysate samples were then subjected to the 3 cycles of freeze thawing. The resulting supernatant was used for DNA quantification and fluorescence signal intensity was detected and recorded between 485-520 nm. Cell viability and total DNA measurement of hES-MP cells of free floating samples were determined using DNA pico-green assay at $24 \mathrm{~h}$, days $6,14,21$ and $28 \mathrm{pc}$.

2.6.3 Statistics: Measurement of microspheres diameter was performed three times using $n=10-12$ collagen beads for each repeat. Visualisation of cell viability at different time points was performed using bright field filter of (Nikon Ti-E) on two or three samples of each condition during experimental repeat. Statistical differences of seeded hES-MPs versus MG-63s microspheres contraction and bovine collagen I contraction against rat tail collagen I was completed using one-way ANOVA with Sidak's and Tukey's post hoc test. Different letters represent significance at $\mathrm{p}<0.05$.

\section{Results}

\subsection{Collagen fibre micro-structure}

SEM microscopy of both seeded and none seeded gel showed a highly porous structure and random pore size with little or no visible aligned fibres in both rat tail and bovine collagen I gels over 21 day pc (Figure 1a-f). The random orientation and mesh work of collagen fibres was confirmed by SHG microscopy. The results from SHG imaging showed no significant difference between microstructure of bovine and rat tail collagen I fibres. Both samples presented amorphous fibrous structures and few visible alignments of collagen fibres, with bovine sample emitting stronger signals and more visibly aligned fibres (Figure 2a-g). 


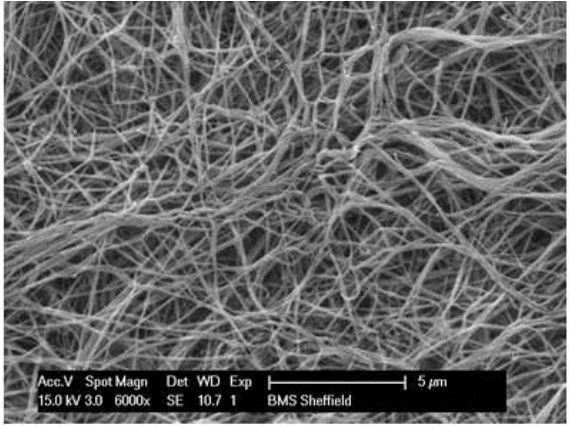

(a)



(c)

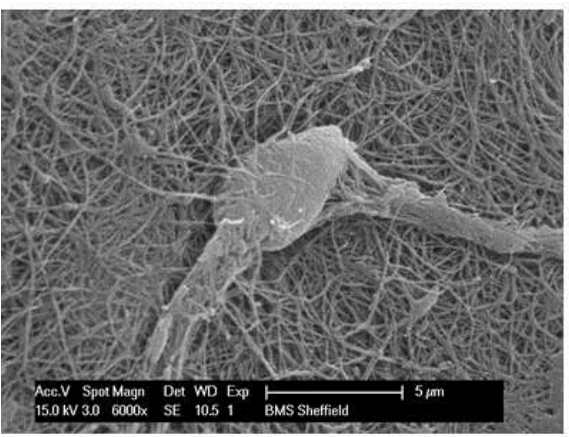

(e)



(b)

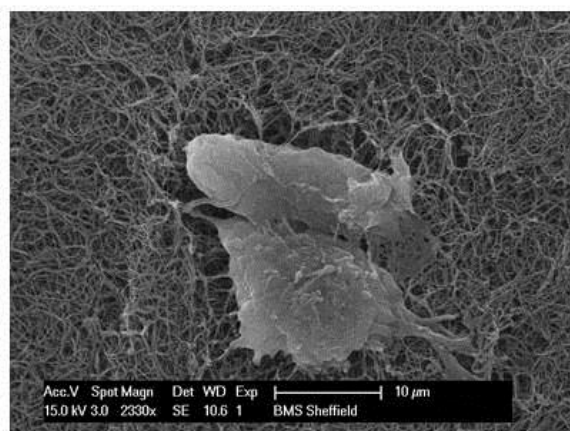

(d)

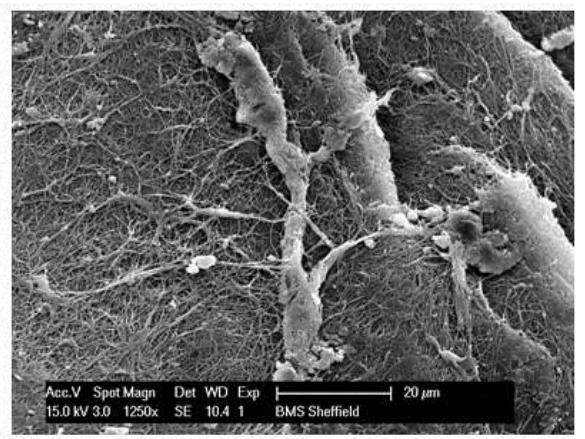

(f)

Figure 1: SEM images of bovine and rat tail collagen I microspheres, micro structure and mesh work of collagen fibres after gelation, (a); cross section of non-seeded rat tail collagen gel at $24 \mathrm{~h} \mathrm{pc}$, (b); cross section of bovine gel at $24 \mathrm{~h},(\mathrm{c}, \mathrm{d})$; hES-MPs seeded rat tail and bovine collagen gel at $24 \mathrm{~h},(\mathrm{e}, \mathrm{f})$; hES-MPs seeded rat tail and bovine collagen gel at day $21 \mathrm{pc}$. Scale bar for $(\mathrm{a}, \mathrm{c}, \mathrm{e})=5 \mu \mathrm{m},(\mathrm{b})=1 \mu \mathrm{m},(\mathrm{d})=10 \mu \mathrm{m},(\mathrm{f})=20 \mu \mathrm{m}$. 


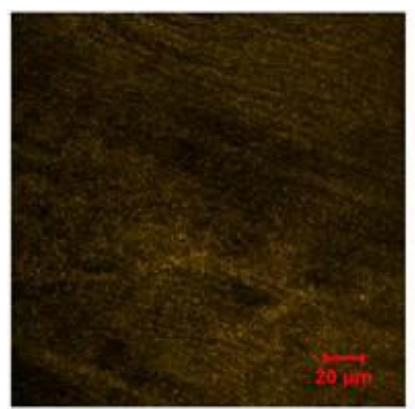

(a)

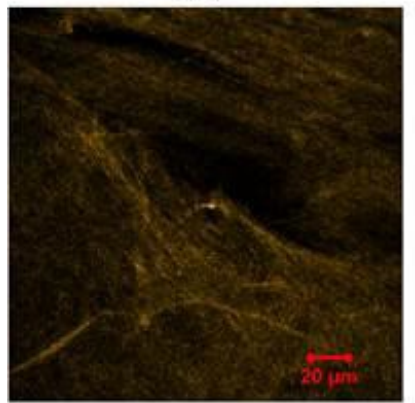

(c)

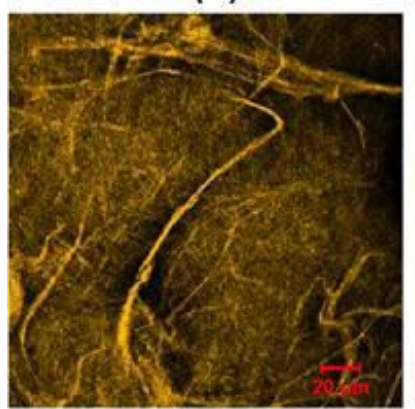

(e)

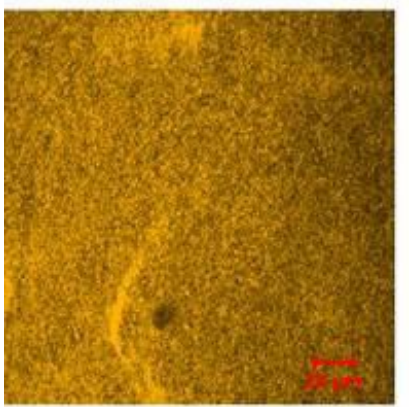

(g)

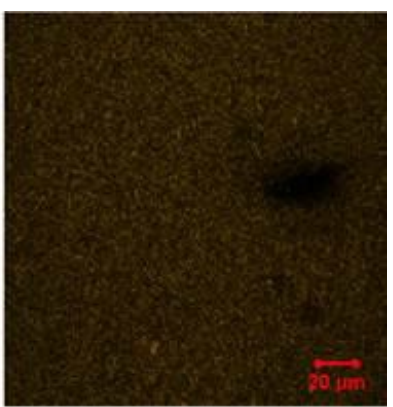

(b)

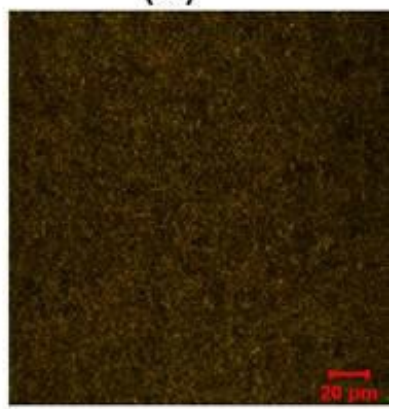

(d)

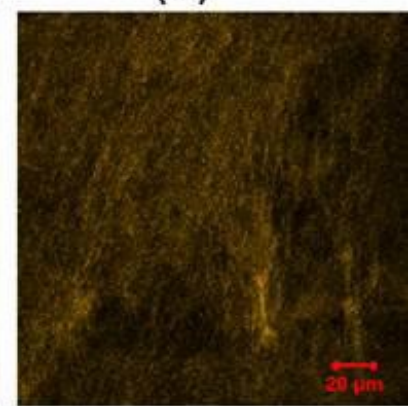

(f)

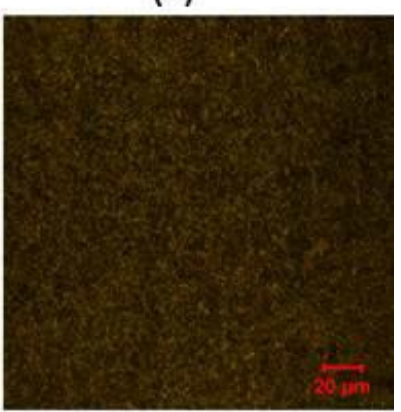

(h)

Figure 2: Microstructure and fibre density of three bovine and rat tail collagen I microspheres were compared using SHG confocal microscope at $24 \mathrm{~h} \mathrm{pc}$. (a, c, e and g); bovine collagen fibres, rat tail collagen at $24 \mathrm{~h} \mathrm{pc},(\mathrm{b}, \mathrm{d}, \mathrm{f}$ and h); rat tail collagen fibres. Bovine collagen fibre presented higher intensity of signals suggesting the presence of more collagen fibres in the samples. Scale bars $=20 \mu \mathrm{m}$. 


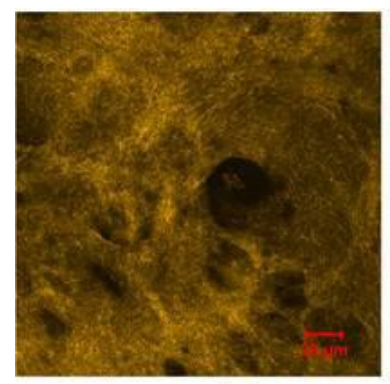

(a)

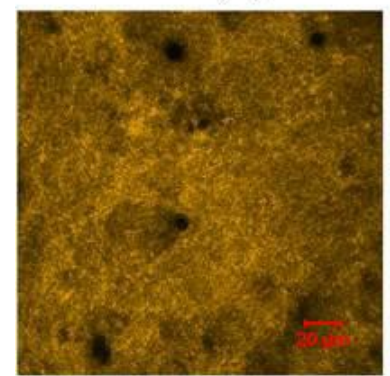

(d)

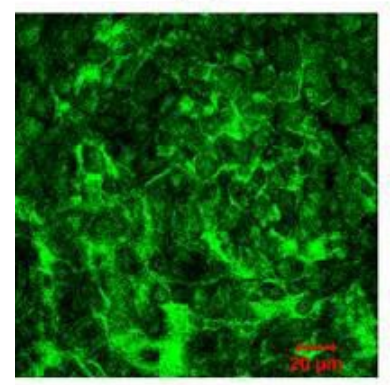

(g)

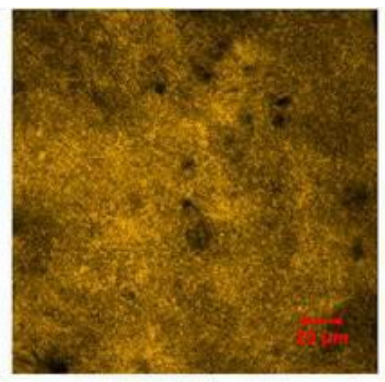

(b)

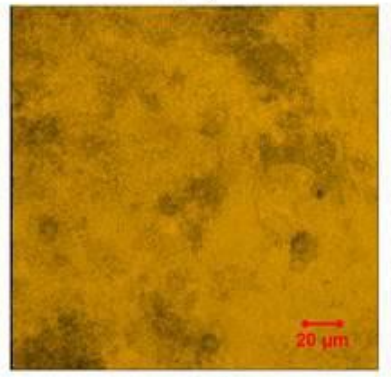

(e)

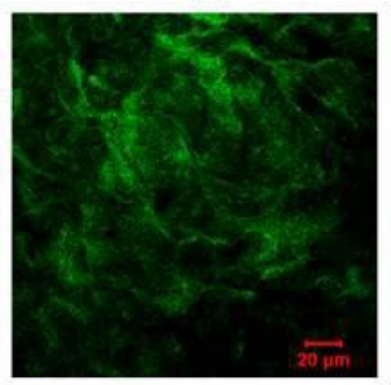

(h)

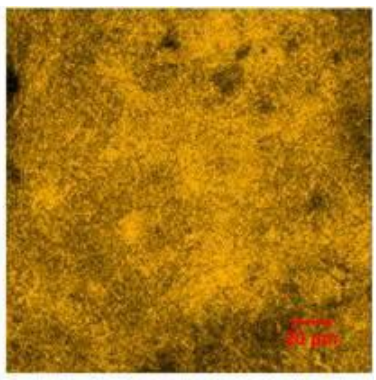

(c)

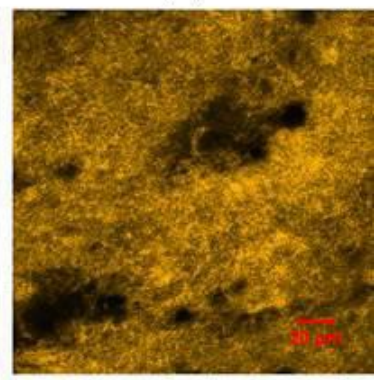

(f)

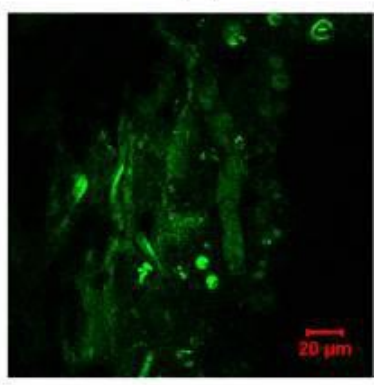

(i)

Figure 3: Microstructure and fibres density of hES-MPs seeded bovine and rat tail collagen I microspheres were compared using SHG confocal microscope on days 7 and $21 \mathrm{pc}$, (a-c); bovine collagen fibre at day $7 \mathrm{pc},(\mathrm{d}-\mathrm{f})$; rat tail collagen fibres at day $7 \mathrm{pc}$, (g-i), bovine collagen fibres at day $21 \mathrm{pc}$. Rat tail collagen fibre presented slightly higher intensity of signals suggesting the presence of more collagen fibres in the samples on day $7 \mathrm{pc}$. Scale bars $=20$ $\mu \mathrm{m}$.

\subsection{Short and long-term cell viability of seeded hES-MPs/collagen microspheres}

hES-MPs with cell density of $5 \times 10^{5}$ cells $/ \mathrm{ml}$ and $1 \times 10^{6}$ cells $/ \mathrm{ml}$ which were embedded in $0.5-2 \mathrm{mg} / \mathrm{mL}$ collagen I gel emitted strong green signal with calcein AM and low red signal with ethidium bromide homodimer at $24 \mathrm{~h} p \mathrm{c}$ (Figure 4a, b, d and f). Also, higher number of elongated cells and better cell interactions were observed when 2 $\mathrm{mg} / \mathrm{mL}$ of bovine collagen I was used. Live cells tended to spread further and migrated to the periphery of collagen beads $48 \mathrm{~h}$ after seeding (Figure $5 \mathrm{c}-\mathrm{g}$ ). 


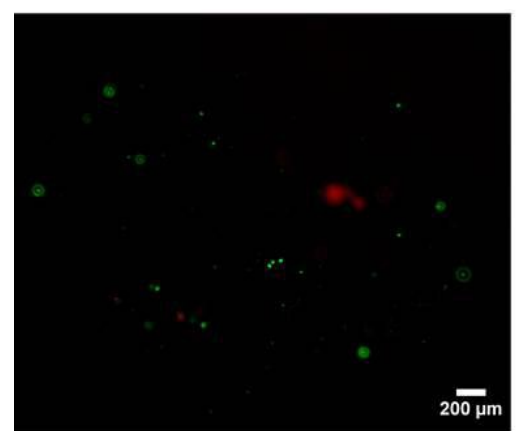

(a)

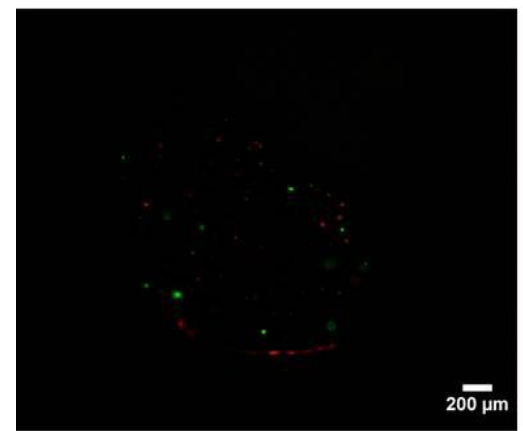

(c)

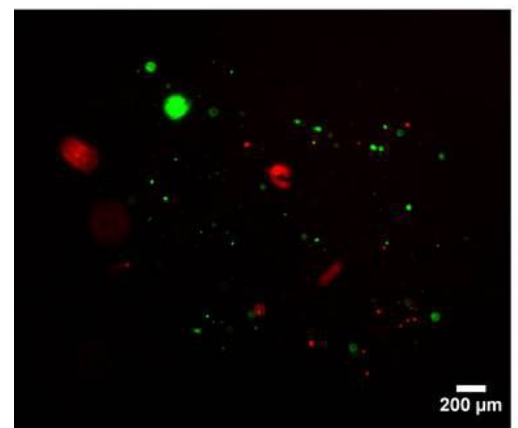

(e)



(b)

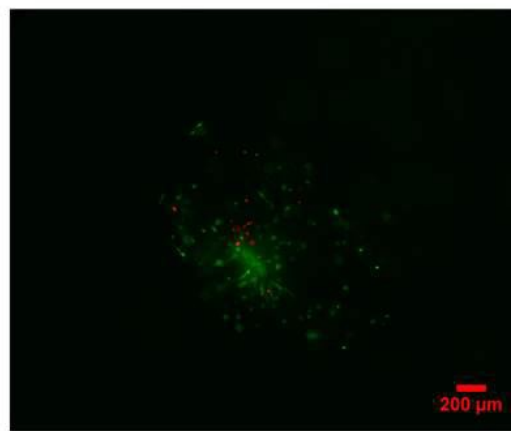

(d)

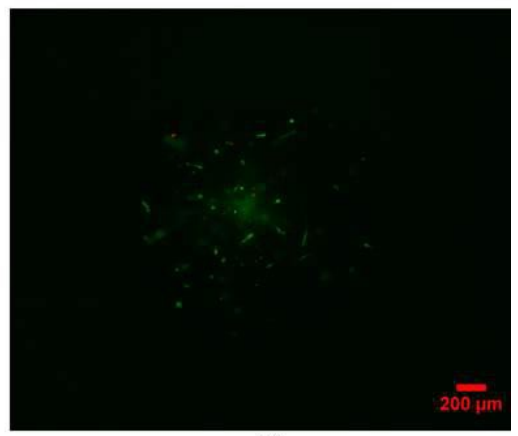

(f)

Figure 4: Effect of collagen concentration and cell seeding density on viability of hES-MPs seeded bovine collagen I microspheres at $24 \mathrm{~h} \mathrm{pc}$. (a); $1 \times 10^{6}$ cells $/ \mathrm{mL}$, collagen concentration (conc): $0: 5 \mathrm{mg} / \mathrm{mL}$ (b); $1 \times 10^{6}$ cells $/ \mathrm{mL}$, collagen conc: $1 \mathrm{mg} / \mathrm{mL}$, (c); $1 \times 10^{6}$ cells $/ \mathrm{mL}$, collagen conc: $3 \mathrm{mg} / \mathrm{mL}$, (d) $1 \times 10^{6}$ cells $/ \mathrm{mL}, \mathrm{c}: 2 \mathrm{mg} / \mathrm{mL}$, (e); $5 \times$ $10^{6}$ cells $/ \mathrm{mL}, \mathrm{c}: 2 \mathrm{mg} / \mathrm{mL},(\mathrm{f}) ; 5 \times 10^{5}$ cells $/ \mathrm{mL}, \mathrm{c}: 2 \mathrm{mg} / \mathrm{mL}$. Scale bars $=200 \mu \mathrm{m}$.

Regardless of cell seeding density, very weak or no green fluorescent signal was detected $24 \mathrm{~h} \mathrm{pc} \mathrm{hES-MP}$ cells in $0.5-1 \mathrm{mg} / \mathrm{mL}$ and $3 \mathrm{mg} / \mathrm{mL}$ collagen and cells shape mainly remained spherical (Figure 4a-b). Cells proliferated steadily till day $35 \mathrm{pc}$ and strong fluorescent signal of elongated viable cells indicated well established cell networks, cell-cell and cell-collagen fibres interactions on the surface of collagen microspheres (Figure $5 \mathrm{f}-\mathrm{g}$ ). 


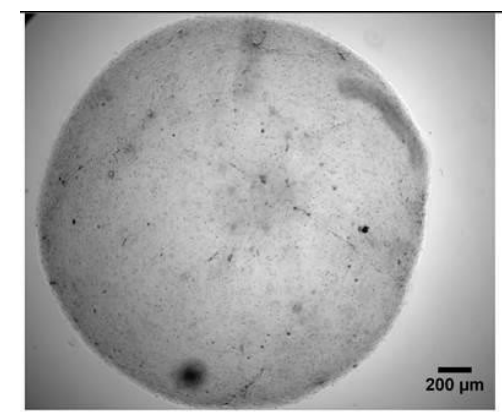

(a)

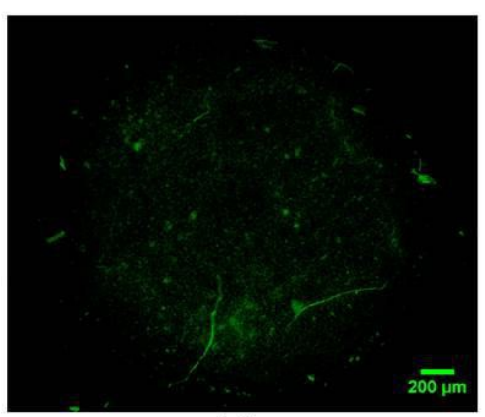

(d)



(b)

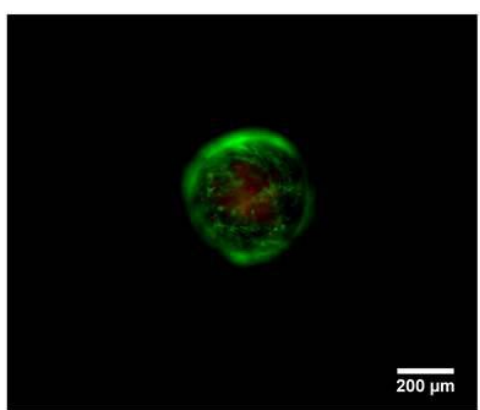

(e)



(c)

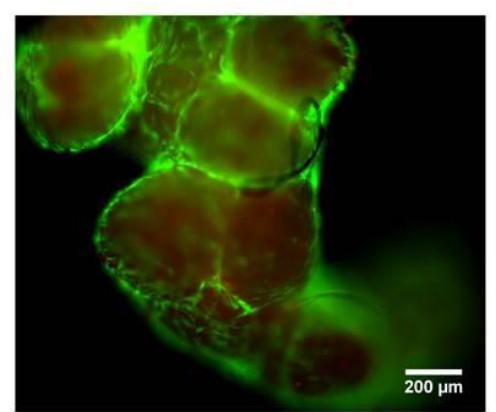

(f)

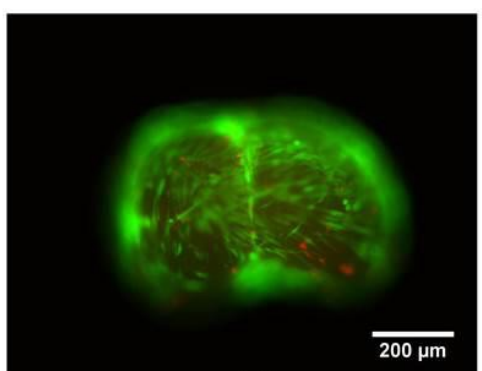

(g)

Figure 5: Short and long-term cell viability of seeded hES-MPs collagen I bovine microsphere were evaluated by bright field and fluorescent imaging of live/dead cells over 35 days pc. (a); 24 h pc, (b) 48 h pc, (c); day 3 pc, (d); day 5 pc, (e); day 7 pc, (f, g); day 35 pc. Live cells were shown by green colour while, dead cells were stained red.

$$
\text { Scale bars }=200 \mu \mathrm{m} \text {. }
$$

Results of DNA pico green assay indicated that the total DNA and cell number remained fairly stable over 28 days pc (Figure 6). The number of cells was reduced by 14\% between day 1 and day 6 before steadily increasing by $6 \%$ on day $14 \mathrm{pc}$. The total DNA number alleviated to around $50 \mathrm{ng}$ and reached the lowest cell number on day 21 . It then surged by $23 \%$ to $65 \mathrm{ng}$ of DNA on day $28 \mathrm{pc}$. No statistical significance was reported for the total DNA number over 28 days experiment. 




Figure 6: hES-MPs cell viability was evaluated over 28 day's pc by using DNA pico green assay at different time points. No statistical difference was reported.

\subsection{Determination of seeded MG-63s collagen I microspheres viability}

MG63 cells seeded in $3 \mathrm{mg} / \mathrm{ml}$ collagen I and at density of $1 \times 10^{6}$ cells $/ \mathrm{mL}$ showed good viability over 21 days pc (Figure 7a-e). Cells elongated well and gathered to make cell clusters as observed on day 21 (Figure 7d-e). Cells did not survived beyond $24 \mathrm{~h}$ pc with lower collagen concentration below $3 \mathrm{mg} / \mathrm{mL}$ and other cell seeding densities.

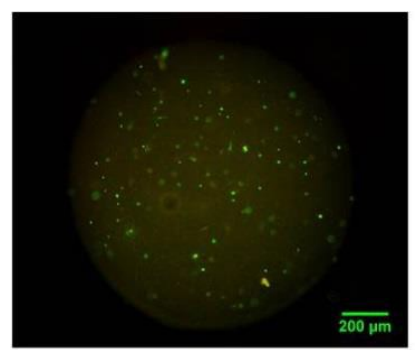

(a)

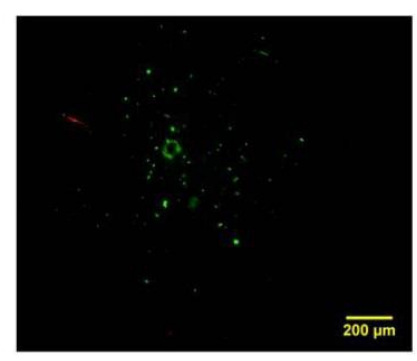

(b)

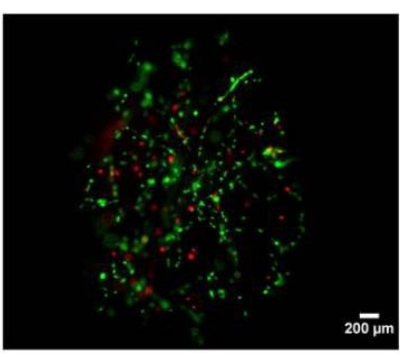

(c)



(d)

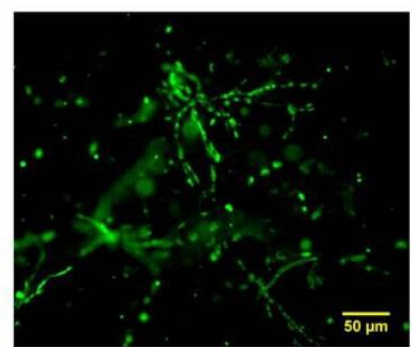

(e)

Figure 7: Long term cell viability of seeded MG-63s collagen I microsphere was checked by fluorescent imaging of live/dead cells over 21 days pc. (a); 24 h pc, (b); day 3 pc, (c); day 5 pc, (d, e); day 21 pc. Collagen conc =2 mg/mL. Scale bars for (a - d) are $200 \mu \mathrm{m}$ and (e) $50 \mu \mathrm{m}$.

\subsection{Collagen gel contraction}

Monitoring hES-MPs/collagen beads showed that collagen microspheres with collagen concentration of $2 \mathrm{mg} / \mathrm{mL}$ and cell number of $1 \times 10^{6}$ cells $/ \mathrm{mL}$ significantly contracted by an average of $43 \%$ within 7 days pc, while the 
contraction remained stable between day 7 and 11 pc (Figure 8a). Average diameter reduction of 20\% was reported between day 3 and day 5, while least contraction with only 14\% change in diameter was observed between day 0 and $3 \mathrm{pc}$. The biggest drop in microsphere diameter and most substantial contraction was reported between day 3 and 7 pc. In contrast, seeded MG-63 cells in collagen microspheres contracted up to $15 \%$ over 7 days of experiment. Collagen microspheres contracted by $10 \%$ within $8 \mathrm{~h}$ while most significant contraction was $12 \%$ between day 2 and 7 of the experiment (Figure 8a). Nonetheless, hES-MPs contracted collagen gel considerably more than MG-63 cells and their average diameter on day $7 \mathrm{pc}$ was significantly smaller than MG-63s $(28 \%, p \leq 0.001)$.

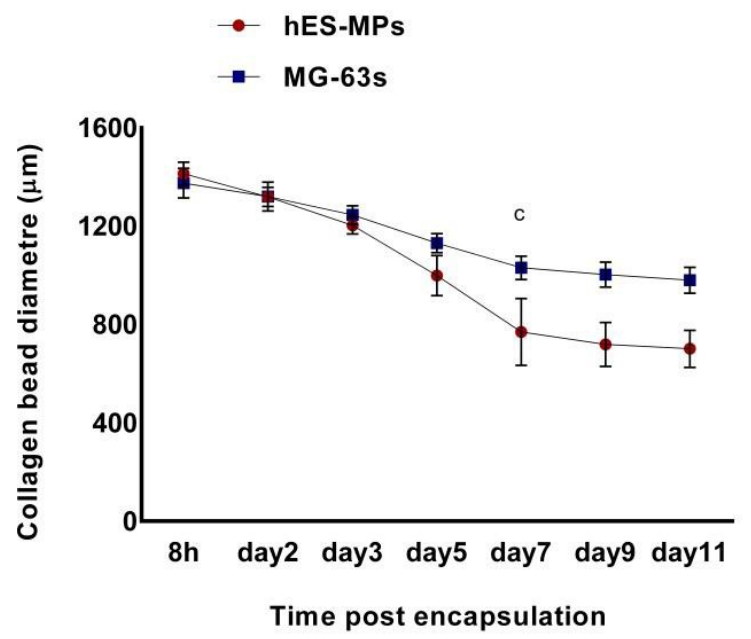

(a)

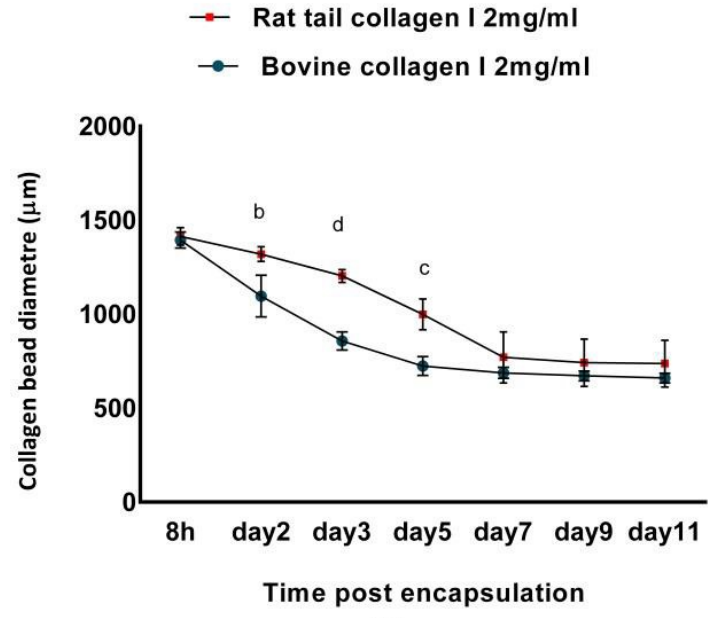

(b)

Figure 8: Effect of two cell lines and collagen I sources on the contraction of collagen I gel with concentration of 2 $\mathrm{mg} / \mathrm{mL}$ were investigated, (a); collagen contractibility of $\mathrm{hES}-\mathrm{MPs} /$ bovine collagen I was compared with rat tail collagen I by measuring the diameter of 12 samples at each time point, (b); ability of hES-MPs versus MG-63 cell line in contracting collagen gel was assessed. ( $\left.{ }^{b} p<0.01,{ }^{c} p<0.001,{ }^{d} p<0.0001\right)$. Data is mean \pm SD $n=9$. 
The results of bovine and rat tail collagen gel contraction revealed that bovine collagen played greater role in supporting hES-MPs collagen contraction (Figure 8b). Bovine collagen microspheres contracted considerably more within the first 3 day pc (39\%), while rat tail collagen gel showed more stable contraction of $15 \%$ in the same period of time. On average rat tail collagen gel contracted 29\% $(p \leq 0.0001)$ less than bovine within 3 day of gelation but in the second half of experiment the average diameter of rat tail collagen microsphere dropped significantly by $35 \%$ compared to $20 \%$ decrease in bovine sample to reach around $700 \mu \mathrm{m}$ on day $7 \mathrm{pc}$.

\section{Discussion}

This study showed that undifferentiated and differentiated cells respond differently to stiffness of their environment as progenitor cells proliferated more and spread on lower concentration of rat tail collagen I gel $(1.5-2 \mathrm{mg} / \mathrm{mL})$ while more differentiated cells (MG-63s) adapted better to a stiffer environment ( $3 \mathrm{mg} / \mathrm{mL}$ ) with decreased viability on softer surfaces. These results are in agreement with Chan et al. [11] and Hong et al. [15] who showed that collagen concentration of $2 \mathrm{mg} / \mathrm{mL}$ and $3.5 \mathrm{mg} / \mathrm{mL}$ support proliferation of MSCs and human breast cancer cell line MDA-MB-231 respectively.

Cell seeding density was shown to be critical in survival and proliferation of encapsulated cells. Seeding low cell number indicated reduced cell viability within the first $24 \mathrm{~h}$ of encapsulation in both cell lines. Concentration of $1 \times$ $10^{6}$ cells $/ \mathrm{mL}$ showed the highest cell viability and number over 7 days pc in both hES-MPs and MG-63 cell lines. These findings are in line with Chan et al. [11] who showed optimum cell viability with cell density of $1 \times 10^{6}$ cells $/ \mathrm{mL}$.

Encapsulated hES-MPs induced a higher contraction of collagen microspheres than MG-63s. Collagen contraction in both cell lines was most substantial between day 3 and 7 pc, which was in contrast with Chan et al. [16] and Li et al. [17] studies who reported the most significant collagen contraction within the first $30 \mathrm{~h} \mathrm{pc}$. Furthermore, seeded hES-MPs proliferation results presented a delayed in cell growth between day 1 and $5 \mathrm{pc}$ that was followed by a steady increase in cell number. This finding suggests that hES-MPs remodel and reorganise their new environment before spreading and proliferation. The synergy between decreasing the cell number and no notable contraction confirmed cell contraction is vital for their existence, elongation and proliferation in the new soft micro environment.

More elongated cell morphology was observed in MG-63s compared to hES-MPs. One possible explanation would be terminally differentiated MG-63s selectively proliferated on higher stiffness of collagen scaffold that applied more local strains to the cells compared with hES-MPs less stiff environment. Both cell lines showed enhanced cellcell networks in microspheres peripheries 3 days after seeding and cell clustering was observed from day $21 \mathrm{pc}$ onwards.

Cell density seemed to be more important than collagen concentration in microsphere contraction. As results indicated, collagen droplets with cell seeding density of 1,200 cells $/ 5 \mu \mathrm{L}$ droplet and collagen concentration of 2 
$\mathrm{mg} / \mathrm{mL}$ contracted up to $15 \%$ within the first $30 \mathrm{~h}$ of gelation while droplets with lower cell number showed a delayed ( $96 \mathrm{~h} \mathrm{pc}$ ) and less significant contraction ( 750 cells / $5 \mu \mathrm{L}$ droplet, $8 \%$ ).

Comparison between bovine and rat tail collagen I fibres micro structure presented close similarities between two species. Both hES-MPs seeded collagen microspheres showed more amorphous structure and less organised collagen fibres compared to seeded samples. hES-MPs were added before polymerisation of collagen to the master mix which suggests that cells possibly digested and reorganised collagen fibres in a more random manner for their growth and viability. Nonetheless, stiffness of cell micro-environment cannot provide all the necessary signals for full osteogenic commitment of hES-MPs and the role of other factors such as surface topography, porosity, mechanical and biochemical stimulations should also be considered to draw a more detailed and accurate map for interaction of cells with their environment.

The results of DNA assay and fluorescent staining of the seeded hES-MPs microspheres confirmed that collagen can support long term cell viability and proliferation of the cells and cell seeded collagen microspheres can be used a model to study the effect of mechanical stimulation and fluid flow on cells and specific tissue regeneration. Cells in 3D culture sense and respond to their mechanical environment in a complex and dynamic manner and as a result of an integrated effect of the mechanical behaviour of the ECM, the biology of the mechanotransduction, and local gradient of the secreted molecules. While, 2D models continue to offer valuable insights into cytoskeletal mechanics and cellular mechanisms in interacting with their physical microenvironment, more work in $3 \mathrm{D}$ environments is needed to more effectively link those results with in vivo conditions. In addition, optimization of collagen microsphere stability by coating techniques and evaluation of collagen biodegradability are required to increase the collagen beads efficiency in supporting long term growth of stem cells.

\section{Conclusions}

Collagen microspheres can act as an efficient cell supporting system, providing high viability and proliferation efficiency in hES-MPs post encapsulation. Micro encapsulation technique is an efficient approach in fabricating self-assembled collagen/human hES-MPs microspheres that can be used as delivery devices for MSCs. A variety of collagen concentrations and seeding cell densities were examined to achieve the best condition for viability and proliferation of hES-MPs and MG-63 cell lines. Cell/collagen microspheres were built successfully and both cell lines showed good survival and long term growth in their new environment while, hES-MPs collagen microspheres presented significantly higher contraction over MG-63 seeded collagen beads. Self-assembled hES-MPs collagen microspheres present exceptional cell delivery model in bone healing and repair process and in addressing many inadequacies of the existing cell delivery approaches.

\section{Conflict of interest}

The authors declare that they have no competing interests. 


\section{Acknowledgements}

SHG microscopy of samples was carried out by Dr Nicola Green (University of Sheffield) who is gratefully acknowledged. Financial support from the European Research Council (258321) is acknowledged.

\section{References}

1. Nava M, Raimondi M, Pietrabissa R. Controlling self-renewal and differentiation of stem cells via mechanical cues. J Biomed Biotechnol (2012): 797410-797422.

2. Ding S, Schultz PG. A role for chemistry in stem cell biology. Nat Biotechnol 22 (2004): 833-840.

3. Macchiarini $P$, Jungebluth $P$, Go $T$, et al. Clinical transplantation of a tissue-engineered airway. Lancet 372 (2008): 2023-2030.

4. Discher DE, Mooney DJ, Zandstra PW. Growth factors, matrices, and forces combine and control stem cells. Science 324 (2009): 1673-1677.

5. Lee DA, Knight MM, Campbell J, et al. Stem cell mechanobiology. J Cell Biochem 112 (2011): 1-9.

6. Ruszczak Z, Friess W. Collagen as a carrier for on-site delivery of antibacterial drugs. Adv Drug Deliv 55 (2003): 1679-1698.

7. Shih YV, Tseng KF, Lai HY, et al. Matrix stiffness regulation of integrin-mediated mechanotransduction during osteogenic differentiation of human mesenchymal stem cells. J Bone Miner Res 50 (2011): 730-738.

8. Brouzes E, Medkova M, Savenelli N, et al. Droplet microfluidic technology for single-cell high-throughput screening. PNAS 25 (2009): 195-200.

9. Delaine-smith RM, Reilly GC. The effects of mechanical loading on mesenchymal stem cell differentiation and matrix production. Vitamins \& Hormones 87 (2011): 417-480.

10. Zhang Y, Tang CL, Chen WJ, et al. Dynamic compression combined with exogenous SOX-9 promotes chondrogenesis of adipose-derived mesenchymal stem cells in PLGA scaffold. Eur Rev Med Pharmacol Sci 19 (2015): 2671-2678.

11. Chan BP, Hui TY, Yeung CW, et al. Self-assembled collagen-human mesenchymal stem cell microspheres for regenerative medicine. Biomaterials 28 (2007): 4652-4666.

12. Gasperini L, Mano JF, Reis RL. Natural polymers for the microencapsulation of cells. J R Soc Interface 11 (2014): 20140817.

13. Di Lullo GA, Sweeney SM, Korkko J, et al. Mapping the ligand-binding sites and disease-associated mutations on the most abundant protein in the human, type I collagen. J Biol Chem 277 (2002): 4223-4231.

14. Rosenblatt J, Devereux B, Wallace D. Dynamic rheological studies of hydrophobic interactions in injectable collagen bio- materials. J Appl Polym Sci 50 (1993): 953-963.

15. Hong S, Hsu HJ, Kaunas R, et al. Collagen microsphere production on a chip. Lab Chip 12 (2012): 327780.

16. Chan BP, Hui TY, Wong MY, et al. Mesenchymal stem cell-encapsulated collagen microspheres for bone tissue engineering. Tissue Eng Part C 16 (2010): 225-235. 
17. Li C, Chik T, Ngan AHW, et al. Correlation between compositional and mechanical properties of human mesenchymal stem cell-collagen microspheres during chondrogenic differentiation. Tissue Eng Part A 17 (2011): 777-788.

Citation: Shariatzadeh M, Perrault CM, Lacroix D. Stiffness of Cell Micro-Environment Guides Long Term Cell Growth in Cell Seeded Collagen Microspheres. Archives of Clinical and Biomedical Research 2 (2018): 167-182.

(C) This article is an open access article distributed under the terms and conditions of the

